\title{
ARTICLE OPEN Targeting MUC1-C suppresses BCL2A1 in triple-negative breast cancer
}

Masayuki Hiraki ${ }^{1,4}$, Takahiro Maeda ${ }^{1}$, Neha Mehrotra ${ }^{2}$, Caining Jin ${ }^{1}$, Maroof Alam ${ }^{1}$, Audrey Bouillez ${ }^{1}$, Tsuyoshi Hata ${ }^{1}$, Ashujit Tagde ${ }^{1}$, Amy Keating $^{3}$, Surender Kharbanda ${ }^{1}$, Harpal Singh ${ }^{2}$ and Donald Kufe (iD)

B-cell lymphoma 2-related protein $\mathrm{A} 1(\mathrm{BCL} 2 \mathrm{~A} 1)$ is a member of the $\mathrm{BCL}-2$ family of anti-apoptotic proteins that confers resistance to treatment with anti-cancer drugs; however, there are presently no agents that target BCL2A1. The MUC1-C oncoprotein is aberrantly expressed in triple-negative breast cancer (TNBC) cells, induces the epithelial-mesenchymal transition (EMT) and promotes anti-cancer drug resistance. The present study demonstrates that targeting MUC1-C genetically and pharmacologically in TNBC cells results in the downregulation of BCL2A1 expression. The results show that MUC1-C activates the $B C L 2 A 1$ gene by an NFKB p65-mediated mechanism, linking this pathway with the induction of EMT. The MCL-1 anti-apoptotic protein is also of importance for the survival of TNBC cells and is an attractive target for drug development. We found that inhibiting MCL-1 with the highly specific MS1 peptide results in the activation of the MUC1-C $\rightarrow$ NF-KB $\rightarrow B C L 2 A 1$ pathway. In addition, selection of TNBC cells for resistance to $A B T-737$, which inhibits $B C L-2, B C L-x L$ and $B C L-W$ but not MCL-1 or BCL2A1, is associated with the upregulation of MUC1-C and BCL2A1 expression. Targeting MUC1-C in ABT-737-resistant TNBC cells suppresses BCL2A1 and induces death, which is of potential therapeutic importance. These findings indicate that MUC1-C is a target for the treatment of TNBCs unresponsive to agents that inhibit anti-apoptotic members of the BCL-2 family.

Signal Transduction and Targeted Therapy (2018)3:13 https://doi.org/10.1038/s41392-018-0013-x

\section{INTRODUCTION}

Mucin 1 (MUC1) is a heterodimeric protein that is overexpressed in $\sim 90 \%$ of triple-negative breast cancers (TNBCs). ${ }^{1,2}$ The MUC1 transmembrane C-terminal (MUC1-C) subunit functions as an oncoprotein by interacting with diverse kinases and effectors that have been linked to transformation. ${ }^{1,3}$ Along these lines, MUC1-C activates the inflammatory TAK1, TGF- $\beta$-activated kinase 1 (TAK1) $\rightarrow$ IKK $\rightarrow$ NF-KB p65 pathway. ${ }^{4-6}$ The MUC1-C cytoplasmic domain interacts directly with NF-KB p65 and promotes the activation of NF-KB p65 target genes, including MUC1, in an autoinductive loop that increases MUC1-C expression. ${ }^{6}$ Activation of MUC1-C $\rightarrow$ NF-KB p65 signaling is, in turn, associated with induction of the ZEB1 gene, which encodes a transcriptional repressor that suppresses $m i R-200 \mathrm{c}$ and drives the epithelial-mesenchymal transition (EMT). ${ }^{7}$ MUC1-C also contributes to the loss of epithelial cell polarity by (i) ZEB1-mediated downregulation of the CRB3, HUGL2 and PATJ polarity factors and (ii) repression of the $\mathrm{CDH} 1$ gene and thereby the expression of E-cadherin, a key protein for the formation of adherens junctions. $^{7-9}$ EMT is a complex process that involves the recruitment of EMT-inducing factors, such as ZEB1, to key target genes in association with changes in their epigenetic regulation. ${ }^{10}$ In this context, MUC1-C $\rightarrow$ NF- $\mathrm{KB}$ p65 signaling activates transcription of the DNA methyltransferase 1 (DNMT1) and $3 b$ (DNMT3b) genes and induces DNA methylation of the $C D H 1$ promoter, which suppresses E-cadherin expression. ${ }^{11}$ Emerging evidence supports a role for the MUC1-C $\rightarrow$ NF-kB p65 pathway in integrating EMT, epigenetic programming and a gene signature of immune evasion. $^{12,13}$

The EMT program includes the acquisition of mesenchymal and stem cell traits with an increased capacity for survival. ${ }^{10,14}$ In this regard, MUC1-C has been linked to signals that attenuate stressinduced cell death. ${ }^{1,3}$ MUC1-C is transported to the mitochondrial outer membrane, where it blocks the apoptotic response to oxidative stress and DNA damage-induced stress. ${ }^{15-17}$ Mechanistically, MUC1-C binds to the pro-apoptotic BAX protein at the critical $\mathrm{BH} 3$ domain, thereby blocking $\mathrm{BAX}$ dimerization and the BAXmediated release of mitochondrial cytochrome $c .{ }^{18}$ MUC1-C also increases the expression of the anti-apoptotic $B C L-x L$ protein by an NF-KB p65-dependent mechanism ${ }^{6}$ and myeloid cell leukemia-1 (MCL-1) protein, which is a major cause of drug resistance in TNBC cells. ${ }^{19}$ B-cell lymphoma 2-related protein A1 (BCL2A1) is another member of the BCL-2 family of anti-apoptotic proteins that is associated with resistance to chemotherapeutics and targeted agents. $^{20} B C L 2 A 1$ functions as a lineage-specific oncogene by blocking cell death. ${ }^{21,22}$ However, there are presently no effective agents for the treatment of cancers that overexpress BCL2A1.

There is no known relationship between MUC1-C signaling and $B C L 2 A 1$. The present study demonstrates that MUC1-C induces BCL2A1 expression in TNBC cells by an NF-KB p65-dependent

\footnotetext{
${ }^{1}$ Dana-Farber Cancer Institute, Harvard Medical School, Boston, MA, USA; ${ }^{2}$ Center for Biomedical, Indian Institute of Technology, Delhi, India and ${ }^{3}$ Departments of Biology and Biological Engineering, Massachusetts Institute of Technology, Cambridge, MA, USA Correspondence: Donald Kufe (donald_kufe@dfci.harvard.edu)

${ }^{4}$ Present address: Department of Gastrointestinal Surgery, Graduate School of Medicine, Osaka University, Suita, Osaka 565-0871, Japan These authors contributed equally: Masayuki Hiraki, Takahiro Maeda.
} 


\section{a MDA-MB-468/tet-MUC1shRNA}
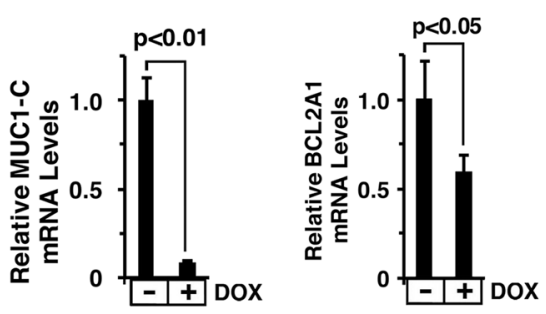

C MDA-MB-468/tet-MUC1shRNA

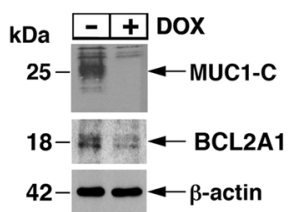

e BT-549/tet-MUC1shRNA

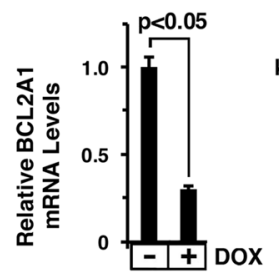

b BT-20/tet-MUC1shRNA

$p<0.01$

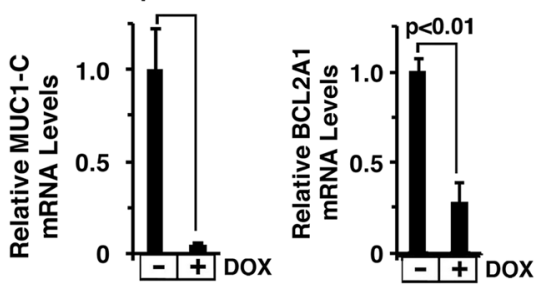

d BT-20/tet-MUC1shRNA

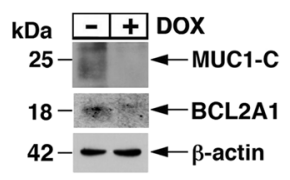

Fig. 1 Downregulation of MUC1-C decreases BCL2A1 expression. a, b MDA-MB-468 (a) and BT-20 (b) cells were transduced to stably express tetracycline-inducible MUC1 shRNA (tet-MUC1shRNA). Cells treated with or without $500 \mathrm{ng} / \mathrm{ml}$ DOX for $3 \mathrm{~d}$ were analyzed for MUC1 (left) and BCL2A1 mRNA levels (right) by qRT-PCR. The results (mean \pm SD of three determinations) are expressed as mRNA levels relative to those in control DOX-untreated cells (assigned a value of 1). c, d Lysates from cells treated with or without $500 \mathrm{ng} / \mathrm{ml}$ DOX for $10 \mathrm{~d}$ were immunoblotted with the indicated antibodies. e BT-549/tet-MUC1shRNA cells treated with or without $500 \mathrm{ng} / \mathrm{ml}$ DOX for $5 \mathrm{~d}$ were analyzed for BCL2A1 mRNA levels by qRT-PCR (left). The results (mean \pm SD of three determinations) are expressed as mRNA levels relative to those in control DOX-untreated cells (assigned a value of 1). Lysates from cells treated with or without $500 \mathrm{ng} / \mathrm{ml} \mathrm{DOX} \mathrm{for} 10 \mathrm{~d}$ were immunoblotted with the indicated antibodies (right)

mechanism. We show that the MUC1-C $\rightarrow$ NF- $\mathrm{KB}$ p $65 \rightarrow \mathrm{BCL} 2 \mathrm{~A} 1$ pathway is of importance in the response to treatment with agents, such as ABT-737, that target other members of the BCL-2 family. In concert with these results, we show that targeting MUC1-C is an effective approach for downregulating BCL2A1 in ABT-737-resistant TNBC cells.

\section{RESULTS}

MUC1-C induces BCL2A1 expression

To assess the potential involvement of MUC1-C in the regulation of BCL2A1, we established MDA-MB-468 breast cancer cells stably expressing tetracycline-inducible control shRNA (tet-CshRNA) or MUC1 shRNA (tet-MUC1shRNA). Treatment of MDA-MB-468/tetMUC1shRNA cells with doxycycline (DOX) was associated with suppression of MUC1-C and BCL2A1 mRNA levels (Fig. 1a, left and right). By contrast, DOX treatment of MDA-MB-468/tet-CshRNA cells had no significant effect on MUC1-C or BCL2A1 expression (Supplemental Fig. S1a, left and right). Similar results were obtained with BT-20 cells stably expressing tet-MUC1shRNA (Fig. 1b, left and right) or tet-CshRNA (Supplemental Fig. S1b, left and right) cells. In concert with these results, DOX treatment of MDA-MB-468/tet-MUC1shRNA (Fig. 1c) and BT-20/tet-MUC1shRNA (Fig. 1d) cells was associated with downregulation of BCL2A1 protein. BT-549/tet-MUC1shRNA cells also responded to DOX by suppressing $B C L 2 A 1$ expression (Fig. 1e, left and right), confirming that MUC1-C drives BCL2A1 expression in TNBC cells.
Targeting MUC1-C suppresses BCL2A1 expression

MUC1-C is a transmembrane protein with a 58-amino acid (aa) extracellular domain and a 72-aa intrinsically disordered cytoplasmic domain ${ }^{23}$ (Fig. 2a). The structure of the MUC1-C subunit is of importance in distinguishing MUC1-C from MUC1$\mathrm{N}$, which is devoid of a transmembrane domain and is shed from the cell surface. The MUC1-C CQC motif in the cytoplasmic domain is necessary for MUC1-C homodimerization and import to the nucleus ${ }^{24,25}$ (Fig. 2a). The MUC1-C cytoplasmic domain also interacts directly with IKK ${ }^{5}$ and NF-KB p65, ${ }^{6}$ thereby activating this inflammatory pathway. To extend the above observations, we stably overexpressed MUC1-C in MDA-MB-468 cells and observed the induction of BCL2A1 mRNA and protein levels (Fig. 2b, left and right). Similar results were obtained in BT20 cells overexpressing MUC1-C (Fig. 2c, left and right), indicating that MUC1-C, and not the shed MUC1-N subunit, is necessary for this response. The GO-203 peptide, which targets the CQC motif and inhibits MUC1-C homodimerization (Fig. 2a), ${ }^{23,25}$ has been incorporated into polymeric nanoparticles (GO-203/NPs) for delivery into tumor cells. ${ }^{26}$ Treatment of MDA-MB-468 cells with GO-203/NPs was associated with the suppression of BCL2A1 mRNA and protein levels compared with empty NPs (Fig. 2d, left and right). We also found that targeting MUC1-C with GO-203/NPs effectively downregulated BCL2A1 expression in BT-20 cells (Fig. 2e, left and right). Of note, GO-203 blocks MUC1-C function ${ }^{1}$ and has no effect on MUC1-C expression in MDA-MB-468 and BT-20 cells. ${ }^{19}$ 
a

MUC1-C

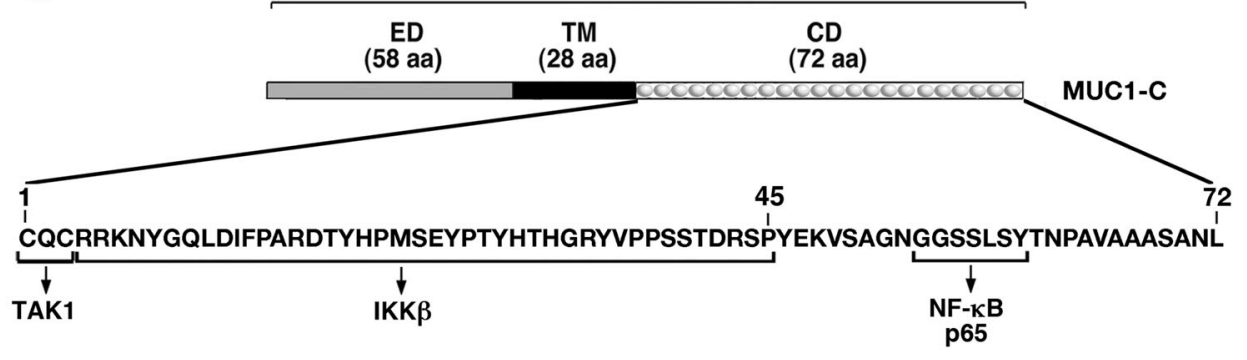

b MDA-MB-468

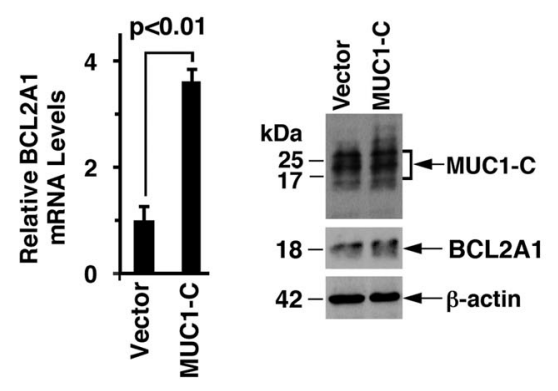

d MDA-MB-468

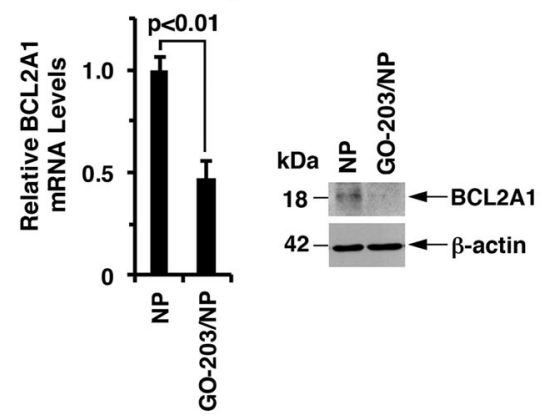

C $\mathrm{BT}-20$

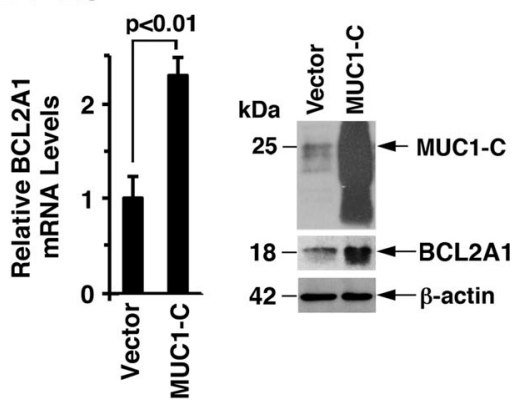

e BT-20

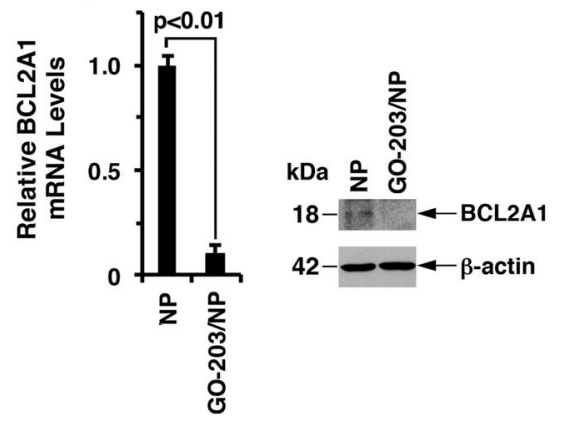

Fig. 2 MUC1-C drives BCL2A1 expression. a Schema of the MUC1-C subunit with the sequence of the 72-amino acid intrinsically disordered cytoplasmic domain (CD). The CQC motif is required for MUC1-C homodimerization and is the target of GO-203. The MUC1-C cytoplasmic domain activates the inflammatory TAK $1 \rightarrow \mid \mathrm{KK} \rightarrow \mathrm{NF}-\mathrm{KB}$ p 65 pathway by direct interactions with these effectors. b, c MDA-MB-468 (b) and BT-20 (c) cells stably transduced to express a control or MUC1-C vector were analyzed for BCL2A1 mRNA levels by qRT-PCR. The results (mean \pm SD of 3 determinations) are expressed as BCL2A1 mRNA levels relative to those in vector cells (assigned a value of 1) (left). Lysates were immunoblotted with the indicated antibodies (right). d, e MDA-MB-468 (d) and BT-20 (e) cells treated with empty NPs or 7.5 $\mu$ M GO-203/NPs for $5 \mathrm{~d}$ were analyzed for BCL2A1 mRNA levels by qRT-PCR. The results (mean \pm SD of three determinations) are expressed as BCL2A1 mRNA levels relative to those in cells treated with empty NPs (assigned a value of 1) (left). Lysates from cells treated with empty NPs or $7.5 \mu \mathrm{M}$ GO-203/NPs for $7 \mathrm{~d}$ were immunoblotted with the indicated antibodies (right)

MUC1-C promotes BCL2A1 transcription by an NF-KB p65mediated mechanism

MUC1-C activates the inflammatory TAK1 $\rightarrow$ IKK $\rightarrow$ NF-KB p65 pathway, binds directly to NF-KB p65 and promotes NF-KB p65-mediated induction of gene transcription. ${ }^{4-6}$ In this way, we found that DOX-induced silencing of MUC1-C in MDA-MB-468/ tet-MUC1shRNA cells decreased phospho-NF-KB p65 ( $p-p 65)$ (Fig. 3a). A similar response was obtained with DOX-treated BT20/tet-MUC1shRNA cells (Supplemental Fig. S2a). The BCL2A1 promoter contains a consensus motif for NF-KB p65 binding (Fig. 3b). ${ }^{27}$ Accordingly, we asked whether silencing NF-KB p65 in MDA-MB-468 cells is associated with the downregulation of $B C L 2 A 1$ transcription. Using this approach, we found that knockdown of NF-KB p65 decreased BCL2A1 mRNA and protein levels (Fig. 3c, left and right). In addition, treatment of MDA-MB468 cells with BAY-11-7085, an inhibitor of IKBa phosphorylation, downregulated $B C L 2 A 1$ expression (Fig. 3d, left and right). Similar results were obtained with BT-20 cells (Supplemental Fig. $\mathrm{S} 2 \mathrm{~b})$. Further supporting the involvement of the MUC1-C $\rightarrow$ NF$\mathrm{KB}$ p $65 \rightarrow \mathrm{BCL} 2 \mathrm{~A} 1$ pathway, MUC1-C silencing was associated with a decrease in NF-кB p65 occupancy at the $B C L 2 A 1$ promoter (Fig. 3e).

Targeting MCL-1 induces BCL2A1 expression

The above findings, taken together with those in our previous work, ${ }^{19}$ support a model in which MUC1-C regulates both MCL-1 and $B C L 2 A 1$. To investigate the relationship between MUC1-Cinduced MCL-1 and BCL2A1 expression, we targeted MCL-1 with the MS1 peptide, which binds to MCL-1 with a dissociation constant of $\sim 2 \mathrm{nM}$ and is $>500$-fold selective for MCL-1 over other BCL-2 family members. ${ }^{28}$ As described for GO-203/NPs, ${ }^{26}$ we encapsulated the MS1 peptide in NPs for intracellular delivery. Treatment of BT-20 and MDA-MB-468 cells with MS1/NPs, but not control NPs, was associated with cell the loss of survival (Fig. $4 a$ and Supplemental Fig. S3). MCL-1 plays a role in suppressing the generation of reactive oxygen species (ROS). ${ }^{29}$ Treatment with MS1/NPs had little, if any, effect on MCL-1 expression and was associated with the upregulation of MUC1-C, NF-KB p65 and BCL2A1 (Fig. 4b), consistent with the finding that ROS activate the MUC1-C $\rightarrow$ NF-KB p65 pathway. ${ }^{19}$ Additionally, combining MS1/NPs 
a MDA-MB-468/tet-MUC1shRNA

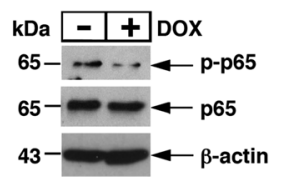

C MDA-MB-468

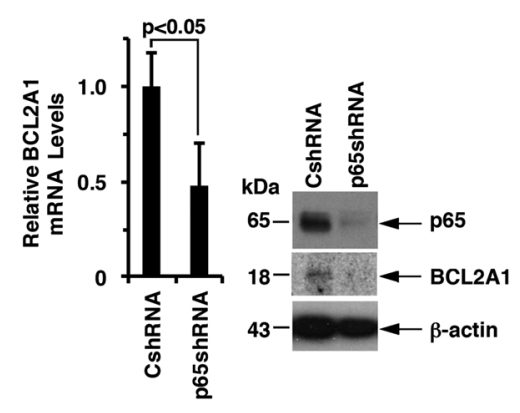

d MDA-MB-468

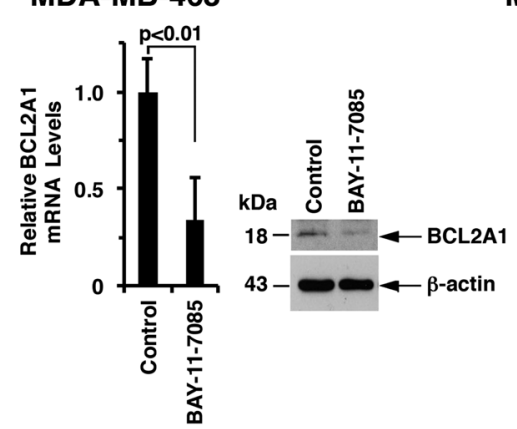

b

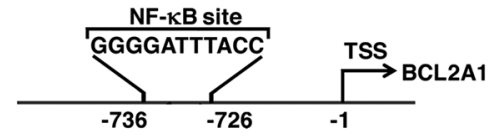

e MDA-MB-468/tet-MUC1shRNA

Fig. 3 MUC1-C $\rightarrow$ NF- $\mathrm{KB}$ p65 signaling induces BCL2A1 expression. a Lysates from MDA-MB-468/tet-MUCshRNA cells treated with or without DOX for $7 \mathrm{~d}$ were immunoblotted with the indicated antibodies. $\mathbf{b}$ Schema of the BCL2A1 promoter with localization of the NF- $\mathrm{KB}$ binding motif at -736 to -726 upstream of the transcription start site (TSS). c MDA-MB-468 cells were stably transduced to express control shRNA (CshRNA) or NF-kB p65 shRNA (p65shRNA). Cells were analyzed for BCL2A1 mRNA levels by qRT-PCR. The results (mean \pm SD of 3 determinations) are expressed as BCL2A1 mRNA levels relative to those in CshRNA cells (assigned a value of 1) (left). Lysates were immunoblotted with the indicated antibodies (right). d MDA-MB-468 cells treated with $5 \mu \mathrm{M}$ BAY-11-7085 or vehicle control for $30 \mathrm{~h}$ were analyzed for BCL2A1 mRNA levels by qRT-PCR. The results (mean \pm SD of three determinations) are expressed as BCL2A1 mRNA levels relative to those in control cells (assigned a value of 1) (left). Cell lysates were immunoblotted with the indicated antibodies (right). e Soluble chromatin from MDA-MB-468/tet-MUC1shRNA cells cultured with or without DOX for $5 \mathrm{~d}$ was precipitated with anti-NF- $\mathrm{kB}$ p65 or a control IgG. The final DNA samples were amplified by qPCR with primers targeting the NF- $\mathrm{KB}$ binding region in the BCL2A1 promoter. The results (mean \pm SD of three determinations) are expressed as the relative fold enrichment compared with the lgG control (assigned a value of 1 )

with GO-203/NPs to inhibit MUC1-C signaling abrogated the MS1/ NP-induced increases in NF-KB p65 and BCL2A1 (Fig. 4b), supporting the importance of MUC1-C in linking MCL-1 activity and the MUC1-C $\rightarrow$ NF-KB p65 $\rightarrow$ BCL2A1 axis (Fig. 4b). In concert with our previous findings, ${ }^{19}$ targeting MUC1-C with GO-203/NPs was also associated with the downregulation of MCL-1 expression (Fig. 4b). GO-203/NPs are effective against TNBC xenografts; ${ }^{26}$ however, MS1/NPs have not been evaluated in vivo. Accordingly, we administered MS1/NPs to mice bearing established BT-20 tumors. The results showed that MS1/NP treatment was effective at significantly inhibiting BT-20 tumor growth (Fig. 4C). In addition, targeting MCL-1 in BT-20 tumors was associated with marked increases in BCL2A1 mRNA levels (Fig. 4d) and the upregulation of MUC1-C, NF-KB p65 and BCL2A1 (Fig. 4e), indicating that targeting $M C L-1$ function in the absence of changes in expression induces cell death and compensatory activation of the MUC1-C $\rightarrow$ NF-KB p65 $\rightarrow$ BCL2A1 pathway.

Resistance to $A B T-737$ and $A B T-263$ is associated with activation of the MUC1-C $\rightarrow$ NF- $\mathrm{kB} \rightarrow \mathrm{BCL} 2 \mathrm{~A} 1$ pathway

The small molecules ABT-737 and ABT-263 target BCL-2, BCL-xL and $B C L-W$ but not MCL-1 or BCL2A1. ${ }^{30,31}$ Selection of $B T-20$ and MDA-MB-468 cells for resistance to ABT-737 (ABT-737R) is associated with the upregulation of MUC1-C and MCL-1. ${ }^{19}$ We also found that BCL2A1 levels were upregulated in BT-20/ABT737R cells (Fig. 5a). In concert with these results, MDA-MB-468 cells resistant to ABT-737 (MDA-MB-468/ABT-737R) or ABT-263 (MDA-MB-468/ABT-263R) also exhibited upregulation of BCL2A1 (Fig. 5b). To determine whether MUC1-C also drives BCL2A1 expression in the setting of ABT-737 resistance, we treated BT-20/ ABT-737R cells with GO-203/NPs. As was reported for MCL-1, ${ }^{19}$ targeting MUC1-C was clearly associated with the downregulation of BCL2A1 expression (Fig. 5c). However, in contrast to MCL-1, which is stabilized by MUC1-C-induced signaling, ${ }^{19}$ targeting MUC1-C in BT-20/ABT-737R cells decreased BCL2A1 mRNA and protein levels (Fig. $5 \mathrm{C}$ ), supporting a potential transcriptional mechanism. Indeed, inhibiting NF-KB p65 with BAY-11-7085 in BT20/ABT-737R (Fig. 5d) and MDA-MB-468/ABT-737R (Fig. 5e) cells effectively suppressed BCL2A1 mRNA levels.

Targeting MUC1-C is effective in the setting of ABT-737 resistance Treatment of BT-20/ABT-737R cells with MS1/NPs was associated with the loss of survival (Fig. 6a). Interestingly, however, MDA-MB468/ABT-737R cells were unaffected by MS1/NP exposure (Fig. 6b), consistent with the finding that BT-20 cells, but not MDA-MB-468 cells, are dependent on MCL-1 for survival. ${ }^{32}$ Notably, in contrast, BT-20/ABT-737R (Fig. 6c) and MDA-MB-468/ABT-737R (Fig. 6d) cells were both sensitive to treatment with GO-203/NPs, supporting our findings that targeting MUC1-C effectively downregulates MCL-1 and BCL2A1.

\section{DISCUSSION}

TNBC is often a clinically aggressive disease. ${ }^{33}$ In addition, options for TNBC treatment have been limited by a lack of actionable molecular targets. ${ }^{33}$ The MUC1-C oncoprotein is aberrantly expressed in most TNBCs $s^{1,2}$ and has been linked to diverse aspects of TNBC progression, including the downregulation of cell polarity factors and the induction of EMT (Fig. 6e). ${ }^{7-9}$ The present study demonstrates that targeting MUC1-C in TNBC cells with genetic and pharmacological approaches suppresses the expression of the anti-apoptotic BCL2A1 protein. BCL2A1 has been identified as an oncogene in melanoma and B-cell malignancies; ${ }^{21}$ however, there are no reports on a role for $B C L 2 A 1$ in breast cancer cells. In this regard, the upregulation of $B C L 2 A 1$ in breast cancers is associated with a significant decrease in relapse-free survival (Supplemental Fig. S4). Our results show that MUC1-C activates $B C L 2 A 1$ expression by an NF-KB p65-mediated mechanism. In this way, MUC1-C binds directly to NF-KB p65 and activates NF-KB target genes, including $B C L-x L$ and $M U C 1$ itself. $^{6}$ By 


\section{b BT-20}

a BT-20

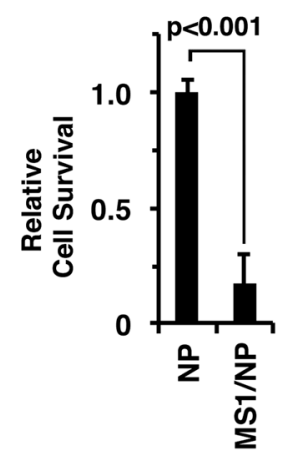

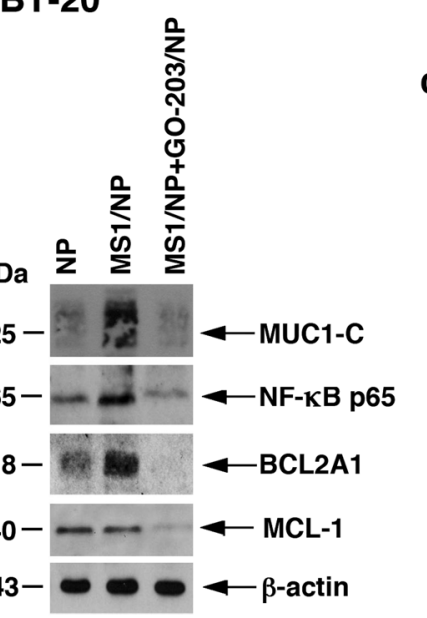

d

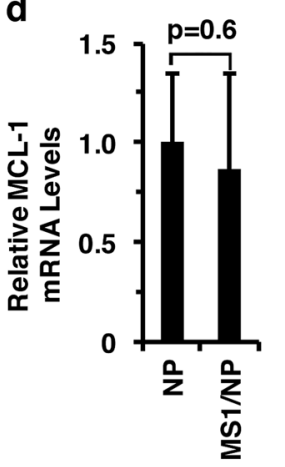

\section{BT-20 Tumors}

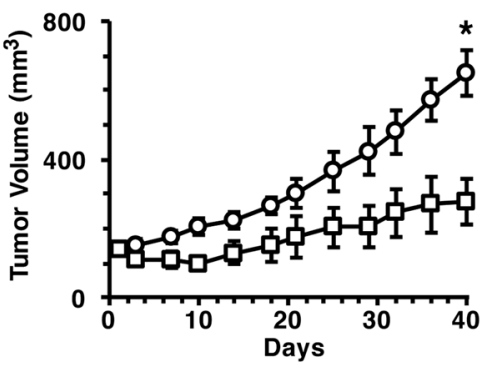

e

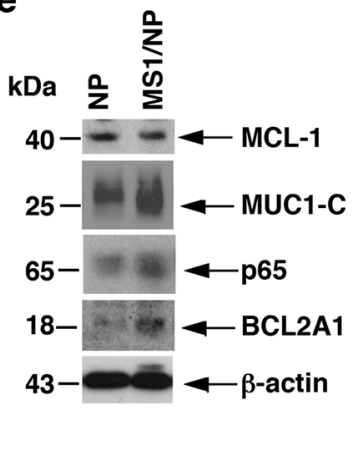

Fig. 4 Targeting MCL-1 activates the MUC1-C $\rightarrow$ NF- $\kappa B$ p65 $\rightarrow$ BCL2A1 pathway. a BT-20 cells were treated with empty NPs or $7.5 \mu M$ MS1/NPs for $5 \mathrm{~d}$. The results are expressed as the relative survival compared with untreated cells (assigned a value of 1). $\mathbf{b}$ BT-20 cells were treated with (i) empty NPs, (ii) $7.5 \mu \mathrm{M}$ MS1/NPs, or (iii) $3.75 \mu \mathrm{M}$ MS1/NPs and $3.75 \mu \mathrm{M}$ GO-203/NPs for $5 \mathrm{~d}$. Lysates were immunoblotted with the indicated antibodies. c BT-20 cells were injected subcutaneously into the flanks of nude mice. Mice with established tumors were pair-matched and then treated intraperitoneally with empty NPs (circles) or $20 \mathrm{mg} / \mathrm{kg} \mathrm{MS1/NPs} \mathrm{(squares)} \mathrm{each} \mathrm{week} \mathrm{for} 3$ weeks. The results are expressed as the tumor volume (mean \pm SEM; six mice per group). ${ }^{*} p<0.01$. Blinding was not performed. Tumors were collected on day 40 . $\mathbf{d}$ Tumor cells were analyzed for MCL-1 (left) and BCL2A1 (right) mRNA levels by qRT-PCR. The results (mean \pm SD of three determinations) are expressed as the relative mRNA levels compared with empty NP-treated tumors (assigned a value of 1). e Lysates from empty NP- and MS1/NP-treated tumors were immunoblotted with the indicated antibodies

extension, the present results show that MUC1-C similarly increases NF-KB p65 occupancy at the BCL2A1 promoter and upregulates $B C L 2 A 1$ expression (Fig. 6e). Of note, these findings do not exclude a potential role for MUC1-C in the posttranscriptional control of $B C L 2 A 1$ expression, which is at present an undeveloped area of investigation. MUC1-C also induces ZEB1 by increasing NF-KB p65 occupancy at the ZEB1 promoter. ${ }^{6,7}$ In turn, ZEB1 suppresses certain genes, such as miR-200c, that promote polarity and thereby drives EMT. ${ }^{7}$ In addition, the inflammatory MUC1-C $\rightarrow$ NF-KB p65 pathway has been linked to the epigenetic regulation of gene expression in TNBC cells by (i) driving DNMT1 and DNMT3b and, in turn, alterations in DNA methylation patterns, ${ }^{11}$ (ii) upregulating components of the Polycomb Repressive Complex 1 (PRC1) and derepressing HOX genes, ${ }^{34,35}$ and (iii) inducing $E Z H 2$ in association with PRC2 activation and the repression of tumor suppressor genes ${ }^{36,37}$ (Fig. 6e). Previous and present findings support a model in which MUC1-C $\rightarrow$ NF-KB p65 signaling integrates the induction of the antiapoptotic BCL2A1 pathway with EMT and epigenetic reprogramming in TNBC cells (Fig. 6e).

Aberrant expression of MCL-1 is important for TNBC cell survival. ${ }^{32}$ As found here for BCL2A1, targeting MUC1-C in TNBC cells is associated with the downregulation of MCL-1. ${ }^{19}$ However, in contrast to MUC1-C-induced BCL2A1 expression by NF-KB p65, MUC1-C increases MCL-1 levels by activating the PI3K $\rightarrow$ AKT and
MEK $\rightarrow$ ERK pathways, thereby stabilizing MCL- 1 protein. ${ }^{19}$ To the best of our knowledge, there is no known relationship between MCL-1 and BCL2A1; nonetheless, the finding that MUC1-C regulates the expression of both proteins invokes the potential for cross-talk. To search for such evidence, we targeted MCL-1 with the highly potent and specific MS1 peptide, ${ }^{28}$ which was encapsulated in nanoparticles for cell delivery. Interestingly, we found that targeting $\mathrm{MCL}-1$ was associated with the upregulation of MUC1-C, NF-KB p65 and BCL2A1. Moreover, the induction of $B C L 2 A 1$ expression was mediated by a MUC1-C-dependent mechanism. MCL-1 protects TNBC cells from death in response to the $\mathrm{BH} 3$ mimetics ABT-737 and ABT-263. ${ }^{30,31}$ Additionally, the selection of TNBC cells for resistance to ABT-737 and ABT-263 is associated with the upregulation of MUC1-C and thereby MCL-1. ${ }^{19}$ Consistent with the observations that MUC1-C drives both MCL-1 and $\mathrm{BCL} 2 \mathrm{~A} 1$ expression, the present results further demonstrate that MUC1-C induces BCL2A1 expression in the setting of resistance to $A B T-737 / 263$. These findings may have clinical relevance in that certain TNBCs overexpressing MCL-1 and BCL2A1 could exhibit resistance to $M C L-1$ inhibitors that are under development. ${ }^{38-40}$ In this context, we found that BT-20/ABT737R cells, which overexpress MCL-1 and BCL2A1, were sensitive to targeting MCL-1 with MS1/NPs. By contrast, MDA-MB-468/ABT737R cells, which also overexpress MCL-1 and BCL2A1, were resistant to targeting $M C L-1$. This discrepancy in sensitivity to 
a $\mathrm{BT}-20$

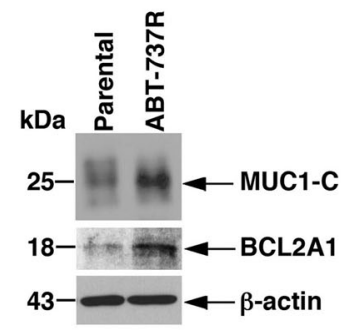

b MDA-MB-468

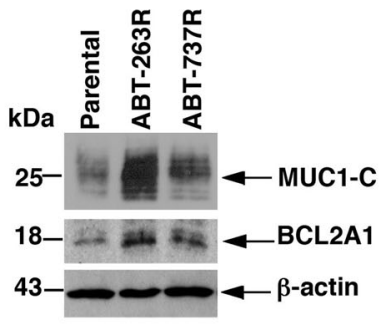

c BT-20/ABT-737R

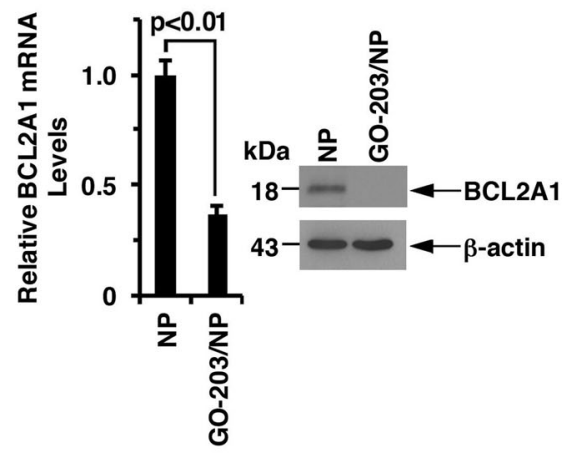

d BT-20/ABT-737R

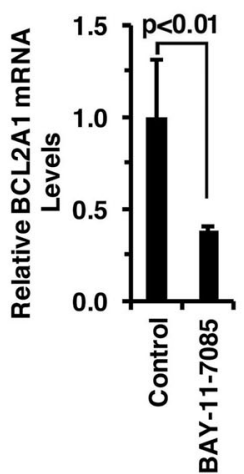

e MDA-MB-468/ABT-737R

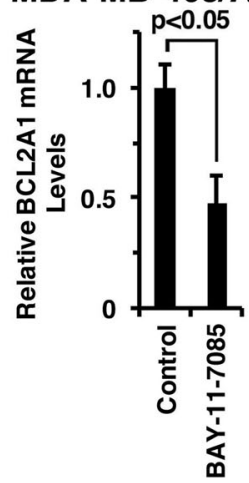

Fig. 5 MUC1-C $\rightarrow$ NF- $\kappa B$ signaling upregulates BCL2A1 in ABT-737-resistant cells. a Lysates from BT-20 (parental) and BT-20/ABT-737R cells were immunoblotted with the indicated antibodies. b Lysates from MDA-MB-468 (parental) and the respective ABT-resistant cells were immunoblotted with the indicated antibodies. c BT-20/ABT-737R cells were treated with empty NPs or 2.5 $\mu \mathrm{M}$ GO-203/NPs for 5 d. Cells were analyzed for BCL2A1 mRNA levels by qRT-PCR. The results (mean \pm SD of three determinations) are expressed as BCL2A1 mRNA levels relative to those in empty NP-treated cells (assigned a value of 1) (left). Lysates were immunoblotted with the indicated antibodies (right). d, e BT-20/ ABT-737R (d) and MDA-MB-468/ABT-737R (e) cells treated with $5 \mu \mathrm{M}$ BAY-11-7085 or vehicle control for $12 \mathrm{~h}$ were analyzed for BCL2A1 mRNA levels by qRT-PCR. The results (mean \pm SD of three determinations) are expressed as BCL2A1 mRNA levels relative to those in control cells (assigned a value of 1)

targeting MCL-1 is explained, at least in part, by the demonstration that BT-20 cells, but not MDA-MB-468 cells, are dependent on MCL-1 for survival. ${ }^{32}$

Our findings further demonstrate that targeting MUC1-C with GO-203/NPs effectively downregulates both MCL-1 ${ }^{19}$ and BCL2A1 in drug-naïve and ABT-737-resistant TNBC cells. In contrast to the differential effects of MS1/NPs, we found that both BT-20/ABT737R and MDA-MB-468/ABT-737R cells were sensitive to targeting MUC1-C, indicating that treatment with GO-203/NPs may be an attractive option in the setting of ABT-737 or ABT-263 resistance. Obatoclax, a pan-BCL-2 family inhibitor that targets $M C L-1$ and $B C L 2 A 1,{ }^{21,41}$ is being evaluated in early-phase clinical trials in combination with cytotoxic and targeted agents (ClinicalTrials. Gov). However, to our knowledge, there are no selective BCL2A1 inhibitors in the clinic. In this context and regarding the translational relevance of the present findings, GO-203 has completed Phase I evaluation in patients with advanced solid tumors, demonstrating an acceptable safety profile and clinical activity. GO-203 has a circulating half-life of 5-7 h, which requires daily drug delivery to maintain necessary tumor exposure levels. To circumvent this challenge, GO-203 has been formulated in NPs for sustained delivery, such that weekly administration of GO-203/ NPs to mice bearing syngeneic or xenograft tumors is as active as daily dosing with GO-203. ${ }^{26}$ Based on the present findings, the GO-203/NP formulation is under development for the treatment of patients with refractory, drug-resistant TNBC.

\section{MATERIALS AND METHODS}

Cell culture

Human MDA-MB-468 cells were grown in Dulbecco's modified Eagle's medium (DMEM) (Corning, Manassas, VA, USA). BT-20 cells were grown in Eagle's Minimum Essential Medium (EMEM) (ATCC, Manassas, VA, USA). BT-549 cells were cultured in RPMI1640 medium (ATCC). Media were supplemented with 10\% heatinactivated fetal bovine serum (HI-FBS), $100 \mathrm{U} / \mathrm{ml}$ penicillin and $100 \mu \mathrm{g} / \mathrm{ml}$ streptomycin. Cells were infected with lentiviral vectors to stably express scrambled control shRNA (CshRNA; Sigma, St. Louis, MO, USA), NF-kB p65 shRNA (Sigma), or MUC1-C. ${ }^{19}$ Cells were treated with (i) the NF-KB inhibitor BAY-11-7085 (Millipore, Billerica, MA, USA) or DMSO as the vehicle control, (ii) the MUC1-C inhibitor GO-203 or the control CP-2 peptide, ${ }^{34}$ or (iii) empty nanoparticles (NPs), GO-203/NPs ${ }^{26}$ or MS1/NPs. ${ }^{28}$ Authentication of the cells was performed by short tandem repeat (STR) analysis. Cells were monitored for mycoplasma contamination using the MycoAlert ${ }^{\circledR}$ Mycoplasma Detection Kit (Lonza, Rockland, MA, USA).

Tetracycline-inducible MUC1 silencing

MUC1shRNA (MISSION shRNA; Sigma, TRCN0000122938), which targets the MUC1-C sequence ACAGACTTCAATAGTATAA, and a control scrambled CshRNA (Sigma) were inserted into the pLKOtet-puro vector (Addgene, Cambridge, MA, USA; Plasmid \#21915). The viral vectors were produced in HEK293T cells as previously described. ${ }^{19,34}$ Cells were selected for growth in $1-3 \mu \mathrm{g} / \mathrm{ml}$ puromycin. Cells were treated with doxycycline (DOX; Sigma).

RNA extraction and real-time quantitative reverse transcription PCR (qRT-PCR)

Total RNA was isolated with Trizol reagent (Invitrogen, Carlsbad, CA, USA) following the manufacturer's protocol. The High Capacity cDNA Reverse Transcription kit (Applied Biosystems, Grand Island, NY, USA) was used to synthesize complementary DNA from $2.0 \mu \mathrm{g}$ of total RNA as described previously. ${ }^{34}$ Power SYBR Green PCR Master Mix (Applied Biosystems) was used with $1 \mu \mathrm{l}$ of diluted 
a $\mathrm{BT}-20 / \mathrm{ABT}-737 \mathrm{R}$

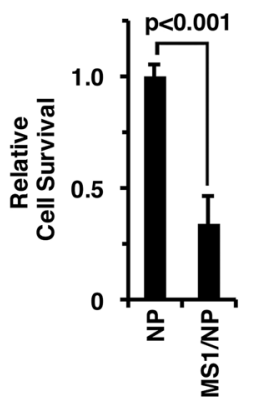

b MDA-MB-468/ABT-737R

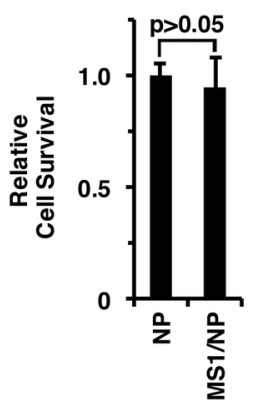

c BT-20/ABT-737R

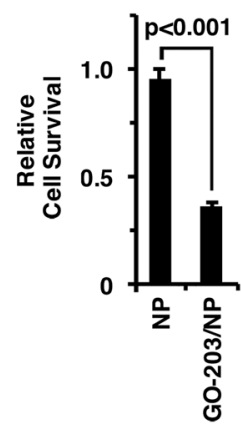

d MDA-MB-468/ABT-737R

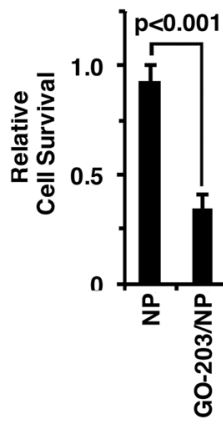

e

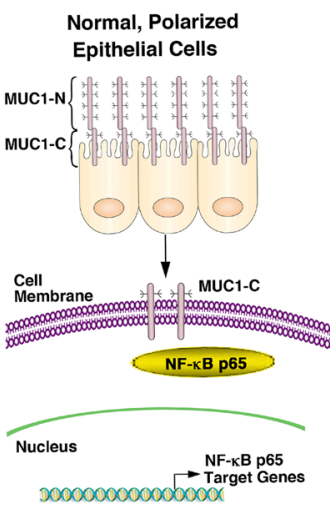

ransformed

Epithelial Cells

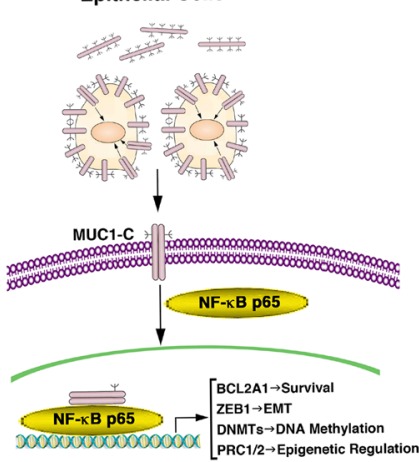

Fig. 6 Targeting MUC1-C is effective against ABT-resistant cells with BCL2A1 overexpression. a, b BT-20/ABT-737R (a) and MDA-MB-468/ ABT-737R (b) cells were treated with empty NPs or $7.5 \mu \mathrm{M}$ MS1/NPs for $7 \mathrm{~d}$. The results are expressed as relative survival compared to untreated cells (assigned a value of 1). c, d BT-20/ABT-737R (c) and MDA-MB-468/ABT-737R (d) cells were treated with empty NPs or 7.5 $\mu \mathrm{M}$ GO-203/NPs for $7 \mathrm{~d}$. The results are expressed as relative survival compared to untreated cells (assigned a value of 1 ). e Schema depicting the function of the MUC1-C $\rightarrow$ NF- $\mathrm{KB}$ p65 pathway in integrating the induction of $B C L 2 A 1$ expression with the EMT program $^{7}$ and epigenetic regulation ${ }^{11,34,36}$

CDNA for each sample. The samples were amplified using the 7300 Real-time PCR System (Applied Biosystems). Primers used for qRT-PCR are listed in Supplemental Table S1.

Chromatin immunoprecipitation (ChIP) assay

Soluble chromatin was isolated from $5 \times 10^{6}$ cells and immunoprecipitated with anti-NF-KB p65 and a control non-immune lgG (Santa Cruz Biotechnology, Dallas, TX, USA). For real-time ChIP qPCR, the SYBR green system was used with the ABI Prism 7300 sequence detector (Applied Biosystems). The data are reported as the relative fold enrichment. ${ }^{11}$ The primers used for ChIP qPCR of the BCL2A1 promoter are listed in Supplementary Table S2.
Immunoblot analysis

Western blot analysis was performed as described previously. ${ }^{34}$ Whole cells were lysed in NP-40 buffer containing a phosphatase inhibitor and protease inhibitor cocktail. Immunoblotting was performed with anti-MUC1-C (NeoMarkers, Fremont, CA, USA), anti-phospho-p65(Ser-536), anti-NF-KB p65 (Santa Cruz Biotechnology), anti-BCL2A1 (Cell Signaling Technology, Danvers, MA, USA) and anti- $\beta$-actin antibodies (Sigma).

Cell survival assays

Cell survival was determined using the Alamar Blue Cell Viability Assay according to the manufacturer's protocol (ThermoFisher Scientific, Waltham, MA, USA).

TNBC tumor xenograft studies

BT-20 cells $\left(5 \times 10^{6}\right)$ were injected subcutaneously into the flanks of 6-week-old female nu/nu mice. After tumors reached $\sim 100 \mathrm{~mm}^{3}$, mice were pair-matched in two groups and treated intraperitoneally with empty NPs or $20 \mathrm{mg} / \mathrm{kg} \mathrm{MS1/NPs} \mathrm{each} \mathrm{week}$ for 3 weeks. Tumor volume was calculated as $V=L \times W^{2} / 2$, where $L$ and $W$ are the larger and smaller diameters, respectively.

Statistical analysis

Each experiment was repeated at least three times. Data are expressed as the mean \pm SD or SEM. The unpaired Student's t-test was used to examine the differences between the means of two groups. A $p$-value $<0.05$ indicated a statistically significant difference.

\section{ACKNOWLEDGEMENTS}

This publication was supported by the US Department of Defense under award number BC151648 and the National Cancer Institute of the National Institutes of Health under award numbers R01 CA097098, R21 CA216553, and R01 CA166480.

\section{ADDITIONAL INFORMATION}

Supplementary information is available for this paper at (https://doi.org/10.1038/ s41392-018-0013-x).

Competing interests: D.K. has equity interests in, serves as a member of the board of directors of and is a paid consultant to Genus Oncology. The remaining authors declare no competing interests.

\section{REFERENCES}

1. Kufe, D. MUC1-C oncoprotein as a target in breast cancer: activation of signaling pathways and therapeutic approaches. Oncogene 32, 1073-1081 (2013).

2. Siroy, A. et al. MUC1 is expressed at high frequency in early-stage basal-like triplenegative breast cancer. Hum. Pathol. 44, 2159-2166 (2013).

3. Kufe, D. Mucins in cancer: function, prognosis and therapy. Nat. Rev. Cancer $\mathbf{9}$, 874-885 (2009).

4. Takahashi, $\mathrm{H}$. et al. MUC1-C activates the TAK1 inflammatory pathway in colon cancer. Oncogene 34, 5187-5197 (2015).

5. Ahmad, R. et al. MUC1 oncoprotein activates the IKB kinase $\beta$ complex and constitutive NF-KB signaling. Nat. Cell Biol. 9, 1419-1427 (2007).

6. Ahmad, R. et al. MUC1-C oncoprotein functions as a direct activator of the NF-KB p65 transcription factor. Cancer Res. 69, 7013-7021 (2009).

7. Rajabi, H. et al. MUC1-C oncoprotein activates the ZEB1/miR-200c regulatory loop and epithelial-mesenchymal transition. Oncogene 33, 1680-1689 (2014).

8. Alam, M., Rajabi, H., Ahmad, R., Jin, C. \& Kufe, D. Targeting the MUC1-C oncoprotein inhibits self-renewal capacity of breast cancer cells. Oncotarget 5, 2622-2634 (2014).

9. Alam, M. et al. MUC1-C represses the Crumbs complex polarity factor CRB3 and downregulates the Hippo pathway. Mol. Cancer Res. 14, 1266-1276 (2016).

10. Tam, W. L. \& Weinberg, R. A. The epigenetics of epithelial-mesenchymal plasticity in cancer. Nat. Med. 19, 1438-1449 (2013).

11. Rajabi, H. et al. DNA methylation by DNMT1 and DNMT3b methyltransferases is driven by the MUC1-C oncoprotein in human carcinoma cells. Oncogene 35, 6439-6445 (2016). 
12. Bouillez, A. et al. MUC1-C integrates PD-L1 induction with repression of immune effectors in non-small cell lung cancer. Oncogene 36, 4037-4046 (2017).

13. Rajabi, H. \& Kufe, D. MUC1-C oncoprotein integrates a program of EMT, epigenetic reprogramming and immune evasion in human carcinomas. Biochim. Biophys. Acta 1868, 117-122 (2017).

14. Polyak, K. \& Weinberg, R. A. Transitions between epithelial and mesenchymal states: acquisition of malignant and stem cell traits. Nat. Rev. Cancer 9, 265-273 (2009).

15. Ren, J. et al. Human MUC1 carcinoma-associated protein confers resistance to genotoxic anti-cancer agents. Cancer Cell 5, 163-175 (2004).

16. Ren, J. et al. MUC1 oncoprotein is targeted to mitochondria by heregulin-induced activation of c-Src and the molecular chaperone HSP90. Oncogene 25, 20-31 (2006).

17. Yin, L. \& Kufe, D. Human MUC1 carcinoma antigen regulates intracellular oxidant levels and the apoptotic response to oxidative stress. J. Biol. Chem. 278, 35458-35464 (2003).

18. Ahmad, R., Alam, M., Rajabi, H. \& Kufe, D. The MUC1-C oncoprotein binds to the $\mathrm{BH} 3$ domain of the proapoptotic BAX protein and blocks BAX function. J. Biol. Chem. 287, 20866-20875 (2012).

19. Hiraki, M. et al. MUC1-C stabilizes MCL-1 in the oxidative stress response of triplenegative breast cancer cells to BCL-2 inhibitors. Sci. Rep. 6, 26643 (2016).

20. Vogler, M. BCL2A1: the underdog in the BCL2 family. Cell Death Differ. 19, 67-74 (2012).

21. Haq, R. et al. BCL2A1 is a lineage-specific antiapoptotic melanoma oncogene that confers resistance to BRAF inhibition. Proc. Natl Acad. Sci. USA 110, 4321-4326 (2013).

22. Sochalska, $M$. et al. MYC selects against reduced BCL2A1/A1 protein expression during B cell lymphomagenesis. Oncogene 36, 2066-2073 (2016).

23. Raina, D. et al. Characterization of the MUC1-C cytoplasmic domain as a cancer target. PLOS ONE 10, e0135156 (2015).

24. Leng, Y. et al. Nuclear import of the MUC1-C oncoprotein is mediated by nucleoporin Nup62. J. Biol. Chem. 282, 19321-19330 (2007).

25. Raina, D. et al. Targeting cysteine-mediated dimerization of the MUC1-C oncoprotein in human cancer cells. Int. J. Oncol. 40, 1643-1649 (2012).

26. Hasegawa, M. et al. Intracellular targeting of the oncogenic MUC1-C protein with a novel GO-203 nanoparticle formulation. Clin. Cancer Res. 21, 2338-2347 (2015).

27. Zong, W. X., Edelstein, L. C., Chen, C., Bash, J. \& Gelinas, C. The prosurvival BCl-2 homolog Bfl-1/A1 is a direct transcriptional target of NF-kappaB that blocks TNFalpha-induced apoptosis. Genes Dev. 13, 382-387 (1999).

28. Foight, G. W., Ryan, J. A., Gulla, S. V., Letai, A. \& Keating, A. E. Designed BH3 peptides with high affinity and specificity for targeting Mcl-1 in cells. ACS Chem. Biol. 9, 1962-1968 (2014).

29. Demelash, A., Pfannenstiel, L. W., Liu, L. \& Gastman, B. R. Mcl-1 regulates reactive oxygen species via NOX4 during chemotherapy-induced senescence. Oncotarget 8, 28154-28168 (2017).
30. Zheng, L. et al. GDC-0941 sensitizes breast cancer to ABT-737 in vitro and in vivo through promoting the degradation of Mcl-1. Cancer Lett. 309, 27-36 (2011).

31. $\mathrm{Wu}, \mathrm{H}$. et al. lonizing radiation sensitizes breast cancer cells to $\mathrm{Bcl}-2$ inhibitor, ABT-737, through regulating Mcl-1. Radiat. Res. 182, 618-625 (2014).

32. Goodwin, C. M., Rossanese, O. W., Olejniczak, E. T. \& Fesik, S. W. Myeloid cell leukemia-1 is an important apoptotic survival factor in triple-negative breast cancer. Cell Death Differ. 22, 2098-2106 (2015).

33. Bianchini, G., Balko, J. M., Mayer, I. A., Sanders, M. E. \& Gianni, L. Triple-negative breast cancer: challenges and opportunities of a heterogeneous disease. Nat. Rev. Clin. Oncol. 13, 674-690 (2016).

34. Hiraki, M. et al. MUC1-C activates BMI1 in human cancer cells. Oncogene 36, 2791-2801 (2016)

35. Tagde, A. et al. Targeting MUC1-C suppresses polycomb repressive complex 1 in multiple myeloma. Oncotarget 8, 69237-69249 (2017).

36. Rajabi, H. et al. MUC1-C activates EZH2 expression and function in human cancer cells. Sci. Rep. 7, 7481 (2017).

37. Rajabi, H., Hiraki, M., Kufe, D. (2018) MUC1-C activates polycomb complexes and downregulates tumor suppressor genes in human cancer cells. Oncogene (Epub ahead of print).

38. Pelz, N. F. et al. Discovery of 2-indole-acylsulfonamide myeloid cell leukemia 1 (Mcl-1) inhibitors using fragment-based methods. J. Med. Chem. 59, 2054-2066 (2016).

39. Rezaei Araghi, R., Ryan, J. A., Letai, A. \& Keating, A. E. Rapid optimization of Mcl-1 inhibitors using stapled peptide libraries including non-natural side chains. ACS Chem. Biol. 11, 1238-1244 (2016).

40. Johannes, J. W. et al. Structure based design of non-natural peptidic macrocyclic Mcl-1 inhibitors. ACS Med. Chem. Lett. 8, 239-244 (2017).

41. Delbridge, A. R., Grabow, S., Strasser, A. \& Vaux, D. L. Thirty years of BCL-2: translating cell death discoveries into novel cancer therapies. Nat. Rev. Cancer 16, 99-109 (2016).

(i) Open Access This article is licensed under a Creative Commons adaptation, distribution and reproduction in any medium or format, as long as you give appropriate credit to the original author(s) and the source, provide a link to the Creative Commons license, and indicate if changes were made. The images or other third party material in this article are included in the article's Creative Commons license, unless indicated otherwise in a credit line to the material. If material is not included in the article's Creative Commons license and your intended use is not permitted by statutory regulation or exceeds the permitted use, you will need to obtain permission directly from the copyright holder. To view a copy of this license, visit http://creativecommons. org/licenses/by/4.0\%.

(c) The Author(s) 2018 